IOANNiS K. Argyros (Lawton, OK)

RAMANDEeP BeHL (Pietermaritzburg)

DANIEl GonzÁlez (Quito)

S. S. Motsa (Pietermaritzburg)

\title{
LOCAL CONVERGENCE FOR MULTISTEP HIGH ORDER METHODS UNDER WEAK CONDITIONS
}

Abstract. We present a local convergence analysis for an eighth-order convergent method in order to find a solution of a nonlinear equation in a Banach space setting. In contrast to the earlier studies using hypotheses up to the seventh Fréchet derivative, we use only hypotheses on the first-order Fréchet derivative and Lipschitz constants. This way, we not only expand the applicability of these methods but also propose a computable radius of convergence for these methods. Finally, concrete numerical examples demonstrate that our results apply to nonlinear equations not covered before.

1. Introduction. One of the most basic and important problem in numerical analysis deals with finding a locally unique solution $x^{*}$ of the equation

$$
F(x)=0,
$$

where $F: D \subset \mathbb{X} \rightarrow \mathbb{Y}$ is a Fréchet differentiable operator, $\mathbb{X}, \mathbb{Y}$ are Banach spaces and $D$ is a convex subset of $\mathbb{X}$. Let us also denote by $L(\mathbb{X}, \mathbb{Y})$ the space of bounded linear operators from $\mathbb{X}$ into $\mathbb{Y}$.

Finding $x^{*}$ is important, since numerous problems can be reduced to equation (1.1) using mathematical modeling [4,5, 9, 13, 18, 21, 22. However, it is not always possible to find the solution $x^{*}$ in closed form. Hence, most methods are iterative. The convergence analysis of iterative methods is usu-

2020 Mathematics Subject Classification: 65G99, 65H10, 47J25, 47J05, 65D10, 65D99.

Key words and phrases: iterative method, local convergence, Banach space, Lipschitz constant, order of convergence.

Received 13 June 2018; revised 26 October 2018.

Published online 12 October 2020. 
ally divided into two categories: semi-local and local convergence analysis. The semi-local convergence analysis is, based on the information around an initial point, to give criteria ensuring the convergence of the method, while the local convergence is based on the information around a solution to find estimates of the radii of convergence balls. An important problem in the study of iterative procedures is the region of accessibility. Therefore, it is very important to propose the radius of convergence of iterative methods.

We study the local convergence of the three-step eighth-order convergent method defined for each $n=0,1,2, \ldots$ by

$$
y_{n}=x_{n}-A_{n}^{-1} F\left(x_{n}\right), \quad x_{n+1}=y_{n}-4 B_{n}^{-1} F\left(y_{n}\right),
$$

where $x_{0} \in D$ is an initial point, $A_{n}=A\left(x_{n}\right)=F^{\prime}\left(x_{n}\right), B_{n}=B\left(x_{n}, y_{n}\right)=$ $F^{\prime}\left(x_{n}\right)+Q\left(x_{n}\right)\left(F\left(x_{n}\right)\right)+2 F^{\prime}\left(\frac{x_{n}+y_{n}}{2}\right)+F^{\prime}\left(y_{n}\right)$ and for each $x \in D, Q(x)(\cdot)$ : $\mathbb{Y} \rightarrow L(\mathbb{X}, \mathbb{Y})$. That is, $Q(x)(y)$ is a linear operator for each $x \in D$ and $y \in \mathbb{Y}$. We can also write $Q(x)(y)=Q(x, y)$. A possible choice for $Q$ is

$$
Q(x)(F(x))=F^{\prime}(x) .
$$

Many other choices are possible. Method 1.2 reduces to a fourth-order convergent method studied in $[20]$ in the special case, when $\mathbb{X}=\mathbb{Y}=\mathbb{R}$, and for

$$
Q(x) G(x)=G^{\prime}(x)
$$

where $G(x) \neq 0$ for each $x \in D-\left\{x^{*}\right\}$. Notice that if $G=F$ then (1.4) reduces to 1.3. In this case, the fourth-order convergence was shown in 20 using Taylor series expansions and hypotheses reaching up to the fourthorder derivative of the involved function, although only the first derivative appears in 1.2 . The function $G$ must satisfy some more conditions, to be found in $20 \mid$. The hypotheses on the derivatives of $F$ restrict the applicability of method 1.2. As a motivational example, define $F$ on $\mathbb{X}=\mathbb{Y}=\mathbb{R}$, $D=[-5 / 2,3 / 2]$ by

$$
F(x)= \begin{cases}x^{3} \ln x^{2}+x^{5}-x^{4}, & x \neq 0 \\ 0, & x=0\end{cases}
$$

Then $x^{*}=1$,

$$
\begin{aligned}
F^{\prime}(x) & =3 x^{2} \ln x^{2}+5 x^{4}-4 x^{3}+2 x^{2}, \\
F^{\prime \prime}(x) & =6 x \ln x^{2}+20 x^{3}-12 x^{2}+10 x, \\
F^{\prime \prime \prime}(x) & =6 \ln x^{2}+60 x^{2}-24 x+22 .
\end{aligned}
$$

Obviously the third-order derivative $F^{\prime \prime \prime}(x)$ of the function involved is not bounded on $D$. Notice in particular that there are a plethora of iterative methods for approximating solutions of nonlinear equations $1-22]$. These results show that the initial point should be close to the required solution 
for the convergence of the corresponding methods. But, how close should the initial point be to $x^{*}$ for the convergence to hold? These local results give no information on the radius of the convergence ball for the corresponding method. That is, the initial point is a shot in the dark. Moreover, no computable error bounds on the distances $\left\|x_{n}-x^{*}\right\|$ are provided or any information on the uniqueness of the solution in a neighborhood of $x^{*}$. We address these problems for method $(1.2)$ in the next section.

In the present study, we expand the applicability of method $(1.2)$ by using only hypotheses on the first-order derivative of $F$ and generalized Lipschitz conditions. Moreover, we avoid using Taylor series expansions and use Lipschitz parameters instead. We apply the computational order of convergence (COC) or the approximate computational order (ACOC) to determine the order of the method, which does not require using derivatives of order higher than one (see Remark 2.2(d)). Our computable error bounds $\left\|x_{n}-x^{*}\right\|$, the region of accessibility as well as the uniqueness of the solution depend on Lipschitz-type constants (see Theorem 2.1 and the numerical examples). It is worth noticing that local convergence results can be used to demonstrate the degree of difficulty in choosing the initial points. This choice is difficult in general because of the need of knowing $x^{*}$ at least approximately. However, in Remark 2.2(e), we provide classes of problems where we can find a radius of convergence without actually knowing $x^{*}$ (see also Example 3.2).

The rest of the paper is organized as follows: Section 2 contains the local convergence analysis of method (1.2). Numerical examples appear in Section 3.

2. Local convergence. In this section, we present the local convergence analysis of method (1.2), by using some scalar functions and parameters. Let $w_{0}, v_{0}, w_{1}$ be increasing and continuous functions defined on $[0,+\infty)$ with values in $[0,+\infty)$ and with $w_{0}(0)=0$. Suppose that the equation

$$
p(t)=1
$$

has at least one positive solution, where

$$
p(t)=w_{0}(t)+\int_{0}^{1} v_{0}(\theta t) d \theta w_{1}(t) t .
$$

Denote by $r_{0}$ the smallest such solution. Let $v:\left[0, r_{0}\right) \rightarrow[0,+\infty)$ and $w:\left[0, r_{0}\right) \rightarrow[0,+\infty)$ be increasing and continuous functions with $w(0)=0$. Moreover, define functions $g_{1}, h_{1}, q$ and $h_{q}$ on $\left[0, r_{0}\right)$ by

$$
\begin{aligned}
& g_{1}(t)=\frac{\int_{0}^{1} w((1-\theta) t) d \theta}{1-w_{0}(t)}+\frac{\left(\int_{0}^{1} v(\theta t) d \theta\right)\left(\int_{0}^{1} v_{0}(\theta t) d \theta\right) w_{1}(t) t}{\left(1-w_{0}(t)\right)(1-p(t))}, \\
& h_{1}(t)=g_{1}(t)-1
\end{aligned}
$$




$$
\begin{aligned}
q(t) & =\frac{1}{4}\left(w_{0}(t)+4 \int_{0}^{1} v_{0}(\theta t) d \theta w_{1}(t) t+2 w_{0}\left(\frac{1}{2}\left(1+g_{1}(t)\right) t\right)+w_{0}\left(g_{1}(t) t\right)\right), \\
h_{q}(t) & =q(t)-1 .
\end{aligned}
$$

We have $h_{1}(0)=h_{q}(0)=-1<0$ and $h_{1}(t) \rightarrow+\infty, h_{q}(t) \rightarrow+\infty$ as $t \rightarrow r_{0}^{-}$. Thus, by the mean value theorem, $h_{1}$ and $h_{q}$ have zeros in $\left(0, r_{0}\right)$. Denote by $r_{1}$ and $r_{q}$ the smallest such zero of $h_{1}$ and $h_{q}$, respectively. Furthermore, define functions $g_{2}$ and $h_{2}$ on $\left[0, r_{0}\right)$ by

$$
\begin{aligned}
& g_{2}(t)=\left[1+\frac{\int_{0}^{1} v\left(\theta g_{1}(t) t\right) d \theta}{1-q(t)}\right] g_{1}(t), \\
& h_{2}(t)=g_{2}(t)-1 .
\end{aligned}
$$

Again we have $h_{2}(0)=-1<0$ and $h_{2}(t) \rightarrow+\infty$ as $t \rightarrow r_{q}^{-}$. Let $r_{2}$ be the smallest zero of $h_{2}$ in $\left(0, r_{q}\right)$. Finally, define

$$
r=\min \left\{r_{1}, r_{2}\right\}
$$

Then, for each $t \in[0, r)$,

$$
\begin{aligned}
& 0 \leq p(t)<1 \\
& 0 \leq w_{0}(t)<1 \\
& 0 \leq g_{1}(t)<1 \\
& 0 \leq q(t)<1 \\
& 0 \leq g_{2}(t)<1
\end{aligned}
$$

Let $U(z, \rho), \bar{U}(z, \rho)$, be respectively the open and closed balls in $\mathbb{X}$ with center $z \in \mathbb{X}$ and of radius $\rho>0$.

Next, we present the local convergence analysis of method 1.2 using the preceding notations.

TheOREM 2.1. Let $F: D \subseteq \mathbb{X} \rightarrow \mathbb{Y}$ be a continuously Fréchet differentiable operator. Let functions $v_{0}, v, w_{0}, w, w_{1}$ be as defined previously and let $r_{0}$ be defined by (2.1). Suppose that there exists $x^{*} \in \mathbb{D}$ such that for each $x \in D$,

$$
F\left(x^{*}\right)=0, \quad F^{\prime}\left(x^{*}\right)^{-1} \in L(\mathbb{Y}, \mathbb{X})
$$

and

$$
\| F^{\prime}\left(x^{*}\right)^{-1}\left(F^{\prime}(x)-F^{\prime}\left(x^{*}\right) \| \leq w_{0}\left(\left\|x-x^{*}\right\|\right) .\right.
$$


Moreover, suppose that for each $x, y \in D_{0}:=D \cap U\left(x^{*}, r_{0}\right)$,

$$
\begin{aligned}
& \left\|F^{\prime}\left(x^{*}\right)^{-1}\left(F^{\prime}(x)-F^{\prime}(y)\right)\right\| \leq w(\|x-y\|), \\
& \left\|F^{\prime}(x)\right\| \leq v_{0}\left(\left\|x-x^{*}\right\|\right), \\
& \left\|F^{\prime}\left(x^{*}\right)^{-1} F^{\prime}(x)\right\| \leq v\left(\left\|x-x^{*}\right\|\right), \\
& \left\|F^{\prime}\left(x^{*}\right)^{-1} Q(x)(F(x))\right\| \leq w_{1}\left(\left\|x-x^{*}\right\|\right), \\
& \bar{U}\left(x^{*}, r\right) \subseteq D,
\end{aligned}
$$

where $Q$ is as defined previously and the radius of convergence $r$ is given by (2.2). Then the sequence $\left\{x_{n}\right\}$ generated for $x_{0} \in U\left(x^{*}, r\right)-\left\{x^{*}\right\}$ by method (1.2) is well defined, remains in $U\left(x^{*}, r\right)$ for each $n=0,1,2, \ldots$ and converges to $x^{*}$. Moreover,

$$
\begin{aligned}
\left\|y_{n}-x^{*}\right\| & \leq g_{1}\left(\left\|x_{n}-x^{*}\right\|\right)\left\|x_{n}-x^{*}\right\| \leq\left\|x_{n}-x^{*}\right\|<r, \\
\left\|z_{n}-x^{*}\right\| & \leq g_{2}\left(\left\|x_{n}-x^{*}\right\|\right)\left\|x_{n}-x^{*}\right\| \leq\left\|x_{n}-x^{*}\right\|,
\end{aligned}
$$

where the functions $g_{1}$ and $g_{2}$ are as defined previously. Furthermore, if

$$
\int_{0}^{1} w_{0}(\theta R) d \theta<1 \quad \text { for } R \geq r,
$$

then $x^{*}$ is the only solution of $F(x)=0$ in $D_{1}:=D \cap \bar{U}\left(x^{*}, R\right)$.

Proof. We shall show by induction that the sequence $\left\{x_{n}\right\}$ is well defined in $U\left(x^{*}, r\right)$ and converges to $x^{*}$. From the hypothesis $x_{0} \in U\left(x^{*}, r\right)-\left\{x^{*}\right\}$, (2.1) -2.3 and (2.9), we have

$$
\left\|F^{\prime}\left(x^{*}\right)^{-1}\left(F^{\prime}\left(x_{0}\right)-F^{\prime}\left(x^{*}\right)\right)\right\| \leq w_{0}\left(\left\|x_{0}-x^{*}\right\|\right)<w_{0}(r)<1 .
$$

It follows from (2.18) and the Banach Lemma on invertible operators 4, 5 that $F^{\prime}\left(x_{0}\right)^{-1} \in L(\mathbb{Y}, \mathbb{X})$ is well defined and

$$
\left\|F^{\prime}\left(x_{0}\right)^{-1} F^{\prime}\left(x^{*}\right)\right\| \leq \frac{1}{1-w_{0}\left(\left\|x_{0}-x^{*}\right\|\right)} .
$$

To show that $y_{0}$ is well defined, it suffices by the first substep of method (1.2) that $A_{0}^{-1} \in L(\mathbb{Y}, \mathbb{X})$. Using (2.1), 2.2), 2.4), 2.9) and (2.11), we get

$$
\begin{aligned}
& \left\|F^{\prime}\left(x^{*}\right)^{-1}\left(A_{0}-F^{\prime}\left(x^{*}\right)\right)\right\| \\
& \quad \leq\left\|F^{\prime}\left(x^{*}\right)^{-1}\left(F^{\prime}\left(x_{0}\right)-F^{\prime}\left(x^{*}\right)\right)\right\|+\left\|F\left(x_{0}\right)\right\|\left\|F^{\prime}\left(x^{*}\right)^{-1} Q\left(x_{0}\right)\right\| \\
& \quad \leq w_{0}\left(\left\|x_{0}-x^{*}\right\|\right)+\int_{0}^{1} v_{0}\left(\theta\left\|x_{0}-x^{*}\right\|\right) d \theta w_{1}\left(\left\|x_{0}-x^{*}\right\|\right)\left\|x_{0}-x^{*}\right\| \\
& \quad=p\left(\left\|x_{0}-x^{*}\right\|\right)<p(r)<1,
\end{aligned}
$$

so $A_{0}^{-1} \in L(\mathbb{Y}, \mathbb{X})$ is well defined and

$$
\left\|A_{0}^{-1} F^{\prime}\left(x^{*}\right)\right\| \leq \frac{1}{1-p\left(\left\|x_{0}-x^{*}\right\|\right)} .
$$


In view of the relations 21.2), 2.1), 2.2, 2.5, 2.10, 2.11, 2.18 and $(2.20)$, we get

$$
\begin{aligned}
& \left\|y_{0}-x^{*}\right\| \\
= & \left\|\left(x_{0}-x^{*}-F^{\prime}\left(x_{0}\right)^{-1} F\left(x_{0}\right)\right)+F^{\prime}\left(x_{0}\right)^{-1}\left(A_{0}-F^{\prime}\left(x_{0}\right)\right) A_{0}^{-1} F\left(x_{n}\right)\right\| \\
\leq & \left\|F^{\prime}\left(x_{0}\right)^{-1} F^{\prime}\left(x^{*}\right)\right\| \\
& \quad \times\left\|\int_{0}^{1} F^{\prime}\left(x^{*}\right)^{-1}\left(F^{\prime}\left(x^{*}+\theta\left(x_{0}-x^{*}\right)\right)-F^{\prime}\left(x_{0}\right)\right)\left(x_{0}-x^{*}\right) d \theta\right\| \\
& +\left\|F^{\prime}\left(x_{0}\right)^{-1} F^{\prime}\left(x^{*}\right)\right\|\left\|F^{\prime}\left(x^{*}\right)^{-1}\left(A_{0}-F^{\prime}\left(x_{0}\right)\right)\right\| \\
\leq & \quad \times A_{0}^{-1} F^{\prime}\left(x^{*}\right)\|\| F^{\prime}\left(x^{*}\right)^{-1} F\left(x_{0}\right) \| \\
\leq & \frac{\int_{0}^{1} w\left((1-\theta)\left\|x_{0}-x^{*}\right\|\right) d \theta\left\|x_{0}-x^{*}\right\|}{1-w_{0}\left(\left\|x_{0}-x^{*}\right\|\right)} \\
& +\frac{\int_{0}^{1} v_{0}\left(\theta\left\|x_{0}-x^{*}\right\|\right) d \theta \int_{0}^{1} v\left(\theta\left\|x_{0}-x^{*}\right\|\right) d \theta w_{1}\left(\left\|x_{0}-x^{*}\right\|\right)\left\|x_{0}-x^{*}\right\|^{2}}{\left(1-w_{0}\left(\left\|x_{0}-x^{*}\right\|\right)\right)\left(1-p\left(\left\|x_{0}-x^{*}\right\|\right)\right)} \\
= & g_{1}\left(\left\|x_{0}-x^{*}\right\|\right)\left\|x_{0}-x^{*}\right\| \leq\left\|x_{0}-x^{*}\right\|<r,
\end{aligned}
$$

which shows (2.18) for $n=0$ and $y_{0} \in U\left(x^{*}, r\right)$. Next, we must show that $B_{0}^{-1} \in L(\mathbb{Y}, \mathbb{X})$. By (2.1), 22.2), 2.6), 2.9), 2.11), 2.12) and (2.22), we get in turn

$$
\begin{aligned}
\leq & \frac{1}{4}\left[\left\|F^{\prime}\left(x^{*}\right)^{-1}\left(F^{\prime}\left(x_{0}\right)-F^{\prime}\left(x^{*}\right)\right)\right\|+4\left\|F^{\prime}\left(x^{*}\right)^{-1} Q\left(x_{0}\right)\left(F\left(x_{0}\right)\right)\right\|\right. \\
& \left.+2\left\|F^{\prime}\left(x^{*}\right)^{-1}\left(F^{\prime}\left(\frac{x_{0}+y_{0}}{2}\right)-F^{\prime}\left(x^{*}\right)\right)\right\|+\left\|F^{\prime}\left(x^{*}\right)^{-1}\left(F^{\prime}\left(y_{0}\right)-F^{\prime}\left(x^{*}\right)\right)\right\|\right] \\
\leq & \frac{1}{4}\left(w_{0}\left(\left\|x_{0}-x^{*}\right\|\right)+4 \int_{0}^{1} v_{0}\left(\theta\left\|x_{0}-x^{*}\right\|\right) d \theta w_{1}\left(\left\|x_{0}-x^{*}\right\|\right)\left\|x_{0}-x^{*}\right\|\right. \\
& \left.+2 w_{0}\left(\frac{1}{2}\left(\left\|x_{0}-x^{*}\right\|+\left\|y_{0}-x^{*}\right\|\right)\right)+w_{0}\left(\left\|y_{0}-x^{*}\right\|\right)\right) \\
\leq & q\left(\left\|x_{0}-x^{*}\right\|\right)<q(r)<1,
\end{aligned}
$$

so that $B_{0}^{-1} \in L(\mathbb{Y}, \mathbb{X})$ is well defined by the second substep of method 1.2 and

$$
\left\|B_{0}^{-1} F^{\prime}\left(x^{*}\right)\right\| \leq \frac{1}{4\left(1-q\left(\left\|x_{0}-x^{*}\right\|\right)\right)} .
$$

Then, by the last substep of method (1.2), and by (2.1), 2.2), 2.7), 2.11), 
(2.22) and 2.24, we have

$$
\begin{aligned}
& \quad\left\|x_{1}-x^{*}\right\| \leq\left\|y_{0}-x^{*}\right\|+4\left\|B_{0}^{-1} F^{\prime}\left(x^{*}\right)\right\|\left\|F^{\prime}\left(x^{*}\right)^{-1} F\left(y_{0}\right)\right\| \\
& \leq g_{1}\left(\left\|x_{0}-x^{*}\right\|\right)\left\|x_{0}-x^{*}\right\|+\frac{\int_{0}^{1} v\left(\theta\left\|y_{0}-x^{*}\right\|\right) d \theta g_{1}\left(\left\|x_{0}-x^{*}\right\|\right)\left\|x_{0}-x^{*}\right\|}{1-q\left(\left\|x_{0}-x^{*}\right\|\right)} \\
& =g_{2}\left(\left\|x_{0}-x^{*}\right\|\right)\left\|x_{0}-x^{*}\right\| \leq\left\|x_{0}-x^{*}\right\|<r,
\end{aligned}
$$

which shows 2.16 for $n=0$ and $z_{0} \in U\left(x^{*}, r\right)$. By simply replacing $x_{0}, y_{0}, x_{1}$ by $x_{k}, y_{k}, x_{k+1}$ in the preceding estimates we arrive at 2.15) and 2.16. Then, in view of the estimates

$$
\left\|x_{k+1}-x^{*}\right\| \leq c\left\|x_{k}-x^{*}\right\|<r, \quad c=g_{2}\left(\left\|x_{0}-x^{*}\right\|\right) \in[0,1),
$$

we deduce that $\lim _{k \rightarrow \infty} x_{k}=x^{*}$ and $x_{k+1} \in U\left(x^{*}, r\right)$.

Finally, to show the uniqueness part, let $y^{*} \in D_{1}$ with $F\left(y^{*}\right)=0$. Define $T=\int_{0}^{1} F^{\prime}\left(x^{*}+\theta\left(x^{*}-y^{*}\right)\right) d \theta$. Using 2.5 and 2.12, we get

$$
\left\|F^{\prime}\left(x^{*}\right)^{-1}\left(T-F^{\prime}\left(x^{*}\right)\right)\right\| \leq \int_{0}^{1} w_{0}\left(\theta\left\|y^{*}-x^{*}\right\|\right) d \theta \leq \int_{0}^{1} w_{0}(\theta R) d \theta<1 .
$$

It follows from 2.27 that $T$ is invertible. Then, in view of the identity

$$
0=F\left(x^{*}\right)-F\left(y^{*}\right)=T\left(x^{*}-y^{*}\right),
$$

we conclude that $x^{*}=y^{*}$.

REMARK 2.2. (a) It follows from 2.10 that condition 2.12 can be replaced by

$$
v(t)=1+w_{0}(t) \quad \text { or } \quad v(t)=1+w_{0}\left(r_{0}\right)
$$

since

$$
\begin{aligned}
\| F^{\prime}\left(x^{*}\right)^{-1} & {\left[\left(F^{\prime}(x)-F^{\prime}\left(x^{*}\right)\right)+F^{\prime}\left(x^{*}\right)\right] \| } \\
& =1+\left\|F^{\prime}\left(x^{*}\right)^{-1}\left(F^{\prime}(x)-F^{\prime}\left(x^{*}\right)\right)\right\| \\
& \leq 1+w_{0}\left(\left\|x-x^{*}\right\|\right)=1+w_{0}(t) \text { for }\left\|x-x^{*}\right\| \leq r_{0} .
\end{aligned}
$$

(b) If $w_{0}$ is strictly increasing, then we can choose

$$
r_{0}=w_{0}^{-1}(1)
$$

instead of 2.1.

(c) If $w_{0}, w, v$ are constant functions (the proof of Theorem 2.1 goes through also in this case), then

$$
r_{1}=\frac{2}{2 w_{0}+w}
$$

and

$$
r \leq r_{1}
$$


Therefore, the radius of convergence $r$ cannot be larger than the radius of convergence $r_{1}$ for Newton's method

$$
x_{n+1}=x_{n}-F^{\prime}\left(x_{n}\right)^{-1} F\left(x_{n}\right) .
$$

Notice also that the earlier radius of convergence given independently by Rheinboldt [19] and Traub [22] is

$$
r_{\mathrm{TR}}=\frac{2}{3 w_{1}},
$$

and by Argyros [4,5],

$$
r_{\mathrm{A}}=\frac{2}{2 w_{0}+w_{1}},
$$

where $w_{1}$ is the Lipschitz constant for (2.6) on $D$. But

$$
w \leq w_{1}, \quad w_{0} \leq w_{1}
$$

So

$$
r_{\mathrm{TR}} \leq r_{\mathrm{A}} \leq r_{1}
$$

and

$$
r_{\mathrm{TR}} / r_{\mathrm{A}} \rightarrow 1 / 3 \quad \text { as } w_{0} / w \rightarrow 0 .
$$

The radius of convergence $q$ used in [4] is smaller than the radius $r_{\mathrm{DS}}$ given by Dennis and Schnabel [4],

$$
q<r_{\mathrm{SD}}=\frac{1}{2 w_{1}}<r_{\mathrm{TR}} .
$$

However, $q$ cannot be computed using the Lipschitz constants.

(d) The order of convergence of method (1.2) was shown in [20] using hypotheses on up to the seventh-order derivative of $F$. We have only used hypotheses on the first-order derivative of $F$. The order of convergence can be determined by using the computational order of convergence COC given by

$$
\xi=\lim _{n \rightarrow \infty} \frac{\ln \frac{\left\|x_{n+2}-x^{*}\right\|}{\left\|x_{n+1}-x^{*}\right\|}}{\ln \frac{\left\|x_{n+1}-x^{*}\right\|}{\left\|x_{n}-x^{*}\right\|}},
$$

or the approximate computational order of convergence (ACOC) [14 given by

$$
\xi^{*}=\frac{\ln \frac{\left\|x_{n+2}-x_{n+1}\right\|}{\left\|x_{n+1}-x_{n}\right\|}}{\ln \frac{\left\|x_{n+1}-x_{n}\right\|}{\left\|x_{n}-x_{n-1}\right\|}} \quad \text { for sufficiently large } n,
$$

which do not require higher than first derivatives. The parameter $\xi^{*}$ does not even require the knowledge of $x^{*}$. Notice also that in the case of convergence there exists $k=0,1,2, \ldots$ such that $x_{n}=x_{n+k}=\xi^{*}$. 
(e) The results obtained here can be used for operators $F$ satisfying the autonomous differential equation [4,5] of the form

$$
F^{\prime}(x)=P(F(x))
$$

where $P$ is a known continuous operator. Since $F^{\prime}\left(x^{*}\right)=P\left(F\left(x^{*}\right)\right)=P(0)$, we can apply the results without actually knowing the solution $x^{*}$. As an example let $F(x)=e^{x}-1$. Then we can choose $P(x)=x+1$.

3. Numerical examples and applications. We present some examples pertaining to the theoretical results of Section 2. We use the choice of $Q$ given by (1.3) in all examples, so by 2.12 and $(2.13)$, we can set $w_{1}(t)=v(t)$.

EXAmPle 3.1. Let $\mathbb{X}=\mathbb{Y}=C[0,1]$ and consider the nonlinear integral equation of mixed Hammerstein type [10,13], defined by

$$
x(s)=\int_{0}^{1} G(s, t)\left(x(t)^{3 / 2}+\frac{x(t)^{2}}{2}\right) d t,
$$

where the kernel $G$ is the Green function defined on $[0,1] \times[0,1]$ by

$$
F(s, t)= \begin{cases}(1-s) t, & t \leq s, \\ s(1-t), & s \leq t .\end{cases}
$$

The solution $x^{*}(s)=0$ is the same as the solution of (1.1), where $F: D \subset$ $C[0,1] \rightarrow C[0,1]$ defined by

$$
F(x)(s)=x(s)-\int_{0}^{t} G(s, t)\left(x(t)^{3 / 2}+\frac{x(t)^{2}}{2}\right) d t .
$$

Notice that

$$
\left\|\int_{0}^{t} G(s, t) d t\right\| \leq \frac{1}{8}
$$

Then

$$
F^{\prime}(x) y(s)=y(s)-\int_{0}^{t} G(s, t)\left(\frac{3}{2} x(t)^{1 / 2}+x(t)\right) d t,
$$

so since $F^{\prime}\left(x^{*}(s)\right)=I$, we have

$$
\left\|F^{\prime}\left(x^{*}\right)^{-1}\left(F^{\prime}(x)-F^{\prime}(y)\right)\right\| \leq \frac{1}{8}\left(\frac{3}{2}\|x-y\|^{1 / 2}+\|x-y\|\right) .
$$

Therefore, we can choose

$$
w_{0}(t)=w(t)=\frac{1}{8}\left(\frac{3}{2} t^{1 / 2}+t\right),
$$


and by Remark 2.2(a),

$$
v_{0}(t)=v(t)=1+w_{0}(t) .
$$

The results in 13, 20 cannot be used to solve this problem, since $F^{\prime}$ is not Lipschitz. However, our results do apply. Based on the above choices of functions and (2.2), we get $r=0.00619113$.

EXAmple 3.2. Suppose that the motion of an object in three dimensions is governed by the system of differential equations

$$
\begin{aligned}
& f_{1}^{\prime}(x)-f_{1}(x)-1=0, \\
& f_{2}^{\prime}(y)-(e-1) y-1=0, \\
& f_{3}^{\prime}(z)-1=0,
\end{aligned}
$$

with $x, y, z \in D$ for $f_{1}(0)=f_{2}(0)=f_{3}(0)=0$. Then the solution of the system is given for $w=(x, y, z)^{T}$ by the function $F:=\left(f_{1}, f_{2}, f_{3}\right): D \rightarrow \mathbb{R}^{3}$ defined by

$$
F(v)=\left(e^{x}-1, \frac{e-1}{2} y^{2}+y, z\right)^{T} .
$$

The Fréchet derivative is given by

$$
F^{\prime}(v)=\left[\begin{array}{ccc}
e^{x} & 0 & 0 \\
0 & (e-1) y+1 & 0 \\
0 & 0 & 1
\end{array}\right]
$$

Then $w_{0}(t)=L_{0} t, w(t)=L t$ and $v_{0}(t)=v(t)=M$, where $L_{0}=e-1<$ $L=e^{1 / L_{0}}=1.789572397$ and $M=e^{1 / L_{0}}=1.7896$. Consequently, we get $r=0.0027781$.

EXAmPle 3.3. Let $\mathbb{X}=\mathbb{Y}=C[0,1]$ be the space of continuous functions defined on $[0,1]$, equipped with the max norm. Let $D=\bar{U}(0,1)$. Define $F$ on $D$ for each $x \in D$ by

$$
F(\varphi)(x)=\phi(x)-5 \int_{0}^{1} x \theta \varphi(\theta)^{3} d \theta .
$$

We get

$$
F^{\prime}(\varphi(\xi))(x)=\xi(x)-15 \int_{0}^{1} x \theta \varphi(\theta)^{2} \xi(\theta) d \theta \quad \text { for each } \xi \in \Omega .
$$

Then $x^{*}=0, L_{0}=7.5, L=15$ and $M=2$. Using method 1.2 for $w_{0}(t)=$ $L_{0} t, v_{0}(t)=v(t)=2$ and $w(t)=L t$, we get $r=0.0013404$.

EXAMPLE 3.4. Returning to the motivational example in the introduction of this paper, we have $L=L_{0}=96.662907$ and $M=2$. Using 
method 1.2 for $w_{0}(t)=L_{0} t, v(t)=2, w(t)=L t$ and $v_{0}(t)=6$, we obtain $r=0.001$.

\section{References}

[1] S. Amat, S. Busquier and S. Plaza, Dynamics of the King and Jarratt iterations, Aequationes Math. 69 (2005), 212-223.

[2] S. Amat, S. Busquier, S. Plaza and J. M. Gutiérrez, Geometric constructions of iterative functions to solve nonlinear equations, J. Comput. Appl. Math. 157 (2003), 197-205.

[3] S. Amat, M. A. Hernández and N. Romero, A modified Chebyshev's iterative method with at least sixth order of convergence, Appl. Math. Comput. 206 (2008), 164-174.

[4] I. K. Argyros, Convergence and Application of Newton-type Iterations, Springer, 2008.

[5] I. K. Argyros and S. Hilout, Computational Methods in Nonlinear Analysis, World Sci., 2013.

[6] I. K. Argyros and S. George, Local convergence for some high convergence order Newton-like methods with frozen derivatives, SeMA J. 70 (2015), 47-59.

[7] A. Cordero and J. R. Torregrosa, Variants of Newton's method using fifth-order quadrature formulas, Appl. Math. Comput. 190 (2007), 686-698.

[8] A. Cordero and J. R. Torregrosa, Variants of Newton's method for functions of several variables, Appl. Math. Comput. 183 (2006), 199-208.

[9] A. Cordero, J. R. Torregrosa and M. P. Vassileva, Increasing the order of convergence of iterative schemes for solving nonlinear systems, J. Comput. Appl. Math. 252 (2012), 86-94.

[10] J. A. Ezquerro and M. A. Hernández, New iterations of $R$-order four with reduced computational cost, BIT 49 (2009), 325-342.

[11] J. A. Ezquerro and M. A. Hernández, A uniparametric Halley-type iteration with free second derivative, Int. J. Pure Appl. Math. 6 (2003), 103-114.

[12] J. M. Gutiérrez and M. A. Hernández, Recurrence realtions for the super-Halley method, Comput. Math. Appl. 36 (1998), 1-8.

[13] M. A. Hernández and E. Martínez, On the semilocal convergence of a three steps Newton-type process under mild convergence conditions, Numer. Algorithms 70 (2015), 377-392.

[14] J. Kou, A third-order modification of Newton method for systems of non-linear equations, Appl. Math. Comput. 191 (2007), 117-121.

[15] Á. A. Magreñán and I. K. Argyros, Ball convergence theorems and the convergence planes of an iterative methods for nonlinear equations, SeMA J. 71 (2015), 39-55.

[16] H. Montazeri, F. Soleymani, S. Shateyi and S. S. Motsa, On a new method for computing the numerical solution of systems of nonlinear equations, J. Appl. Math. 2012, art. $751975,15 \mathrm{pp}$.

[17] M. S. Petković, B. Neta, L. Petković and J. Džunič, Multipoint Methods for Solving Nonlinear Equations, Elsevier, 2013.

[18] F. A. Potra and V. Pták, Nondiscrete Induction and Iterative Proceses, Res. Notes Math. 103, Pitman, Boston, MA, 1984.

[19] W. C. Rheinboldt, An adaptive continuation process for solving systems of nonlinear equations, in: Mathematical Models and Numerical Methods (Warszawa, 1975), Banach Center Publ. 3, PWN, Warszawa, 1978, 129-142.

[20] F. A. Shah, M. A. Noor and M. A. Shafiq, Some generalized recurrence relations and iterative methods for nonlinear equations by using decomposition techniques, preprint. 
[21] J. R. Sharma, R. K. Guha and R. Sharma, An efficient fourth-order weighted-Newton method for system of nonlinear equations, Numer. Algorithms 62 (2013), 307-323.

[22] J. F. Traub, Iterative Methods for the Solution of Equations, Prentice-Hall, Englewood Cliffs, NJ, 1964.

Ioannis K. Argyros

Department of Mathematics Sciences

Cameron University

Lawton, OK 73505, U.S.A.

ORCID: 0000-0002-9189-9298

Daniel González (corresponding author)

Escuela de Ciencias Físicas y Matemáticas

Universidad de Las Américas

Quito 170125, Ecuador

ORCID: 0000-0001-5282-7251

E-mail: daniel.gonzalez.sanchez@udla.edu.ec
Ramandeep Behl

School of Mathematics

Statistics and Computer Sciences University of KwaZulu-Natal

Private Bag X01, Scottsville 3209

Pietermaritzburg, South Africa

ORCID: 0000-0003-1505-8945

S. S. Motsa

School of Mathematics

Statistics and Computer Sciences

University of KwaZulu-Natal

Private Bag X01, Scottsville 3209

Pietermaritzburg, South Africa 